\title{
EDITORIAL
}

\section{Collaborations of China with the world in Synbio}

(C) Higher Education Press and Springer-Verlag Berlin Heidelberg 2017

The emerging field of synthetic biology has gained great attractions in recent years, both in China and around the world. As a result, the number of publications in this field has grown exponentially in the past decade. Analysis of this trend can be found in the article "Bibliometrics analysis of synthetic biology based on Web of Science" [1], which is authored in 2015 by researchers from Peking Union Medical College/Chinese Academy of Medical Sciences with support from the Consulting Project of Chinese Academy of Engineering. Just using "synthetic biology" as the "topic" in Web of Science Core Collection, we can get a coarse estimate of the speed of its growth (Fig. 1). Only 56 papers were published in 1998 globally and in 2016 the number reached more than 1000 . In China, only 10 papers were published in 2007 and the numbers reached over 150 each year in 2015 and 2016.

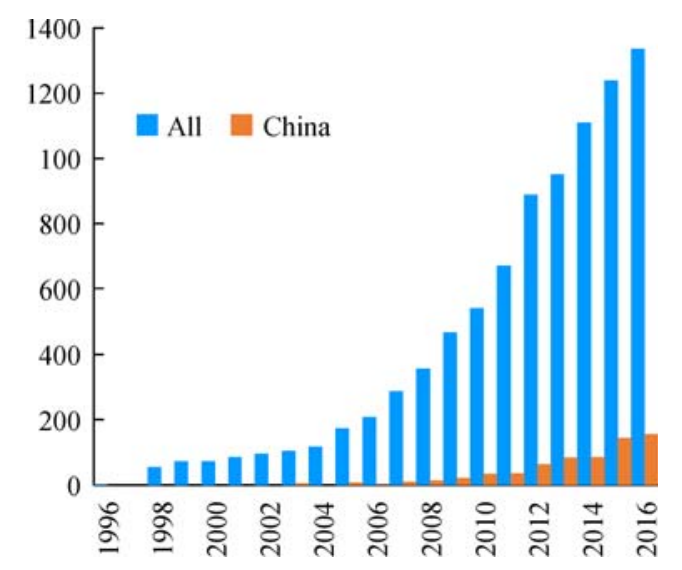

Fig. 1 Numbers of articles published from 1996 to 2016 under topic "synthetic biology". The data were collected from Web of Science Core Collection on 15 February 2017

Definition of synthetic biology has been constantly evolving since its inaugural days. It now includes a wide range of topics, including construction of gene regulatory networks, gene synthesis and genome synthesis, genetic circuit, chassis organisms, minimal genome, and genome engineering. In order to support the growing community and report recent discoveries in this field timely, we were invited as special issue editors of Frontiers of Chemical Science and Engineering to organize this Special Issue: Collaborations of China with the world in Synbio. Seven groups from China, US, Japan, Korea and Singapore made their contributions as Reviews. Most of the research articles were from Chinese groups (6) and one was from Singapore, covering a wide range of topics in synthetic biology. We are also glad to see that professor Taguchi of Hokkaido University gave his view on "Designer enzyme for green materials innovation".

We would like to express our deep appreciation to the members of the editorial office for their outstanding work in planning and organizing to make sure the opportune publication of these articles in Frontiers of Chemical Science and Engineering. We would also like to thank all the authors and reviewers for their contribution and valuable comments. Due to the time limitation, we feel regretful that many excellent research results could not be included in this issue. We could only hope that this compilation can be an inspiration for scientists, especially in China, to motivate continuing efforts in each area of synthetic biology research. 


\section{Yingjin Yuan}

Key Laboratory of Systems Bioengineering (Ministry of Education), School of Chemical Engineering and Technology; SynBio Research Platform, Collaborative Innovation Center of Chemical Science and Engineering (Tianjin), Tianjin University, Tianjin 300092, China

E-mail: yjyuan@tju.edu.cn

Jinchuan $\mathrm{Wu}$

Institute of Chemical and Engineering Sciences, Singapore 627833, Singapore

E-mail: wu_jinchuan@ices.a-star.edu.sg

Xiao Wang

School of Biological and Health Systems Engineering, Arizona State University, Tempe, AZ 85287-9709, USA

E-mail: xiaowang@asu.edu

\section{References}

1. An J L, Tian L, Zhou Y L, Chen D X, Lu Z K. Bibliometrics analysis of synthetic biology based on Web of Science. Progress in Modern Biomedcine, 2015, 15: 139-144 